\title{
Safety and efficacy of recombinant human erythropoietin treatment of anaemia associated with multiple myeloma in haemodialysed patients
}

\author{
P. Ruedin ${ }^{1}$, A. Pechère Bertschi ${ }^{1}$, B. Chapuis ${ }^{2}$, P. Benedet ${ }^{1}$, and M. Leski ${ }^{1}$ \\ Divisions of ${ }^{1}$ Nephrology and ${ }^{2} \mathrm{~d}$ 'Onco-Haematology, Hôpital Cantonal Universitaire, Genève, Switzerland
}

\begin{abstract}
Recombinant human erythropoietin (rHuEpo) was used to treat the anaemia of four haemodialysed patients ( 3 males, 1 female) with advanced multiple myeloma; the type of serum $\mathbf{M}$ component was IgG kappa in all cases. During the 6-month period preceding rHuEpo therapy the patients received multiple blood transfusions (range 4-22 units of packed red cells per patient). After the first month of treatment haematocrit increased from $23 \pm 3$ (SD) to $32 \pm 4 \%$ and during the last 3 months the maintenance dose of rHuEpo was $143 \pm 37 \mathrm{U} / \mathrm{kg}$ per week to achieve a mean haematocrit of $3 \overline{5} \pm 1 \%$. After introduction of rHuEpo, blood transfusions were no longer required and the patients reported an improvement in wellbeing. No apparent worsening of multiple myeloma has been observed over the treatment period ranging from 5 to 34 months (cumulative duration of treatment 55 months). Anti-hypertensive therapy was started in one case and increased in two patients. We conclude that rHuEpo appears to be effective and safe in treating anaemia associated with multiple myeloma in patients requiring haemodialysis.
\end{abstract}

Key words: erythropoietin; anaemia; multiple myeloma; haemodialysis

\section{Introduction}

Recently, recombinant human erythropoietin (rHuEpo) was reported to be effective in treating anaemia associated with multiple myeloma [1]. Nevertheless, its efficacy and tolerance are poorly documented in end-stage renal failure patients with multiple myeloma. In this context the cause of anaemia may be due both to the effects of multiple myeloma on the bone marrow and to the low production of endogenous erythropoietin encountered in end-stage renal disease. Thus it is possible that correction of anaemia in these cases may require larger doses of rHuEpo than in

Correspondence and offprint requests to: Patrick Ruedin MD, Divison de Néphrologie, Département de Médecine, Hôpital Cantonal Universitaire, rue Micheli-du-Crest 24, $\mathrm{CH}-1211$ Genève 14, Switzerland. anaemia due to end-stage renal disease alone. Moreover, the effect of rHuEpo on myeloma growth remains a matter of debate. We report on our experience in $\mathrm{rHuEpo}$ therapy administered in four haemodialysed patients with stage IIIB multiple myeloma IgG kappa; three of them were on maintenance haemodialysis, whereas dialysis was interrupted after 10 weeks in the last patient because of a partial recovery of renal function following chemotherapy.

\section{Subjects and methods}

From August 1988 to June 1991, four haemodialysed patients ( 3 males, 1 female) with multiple myeloma were recruited to receive $\mathrm{rHuEpo}$ treatment for moderate to severe anaemia requiring regular blood transfusions. The aim of the treatment was to achieve a haematocrit around $35 \%$. rHuEpo (Eprex ${ }^{\circledR}$, Cilag, Schaffhausen, Switzerland, in three patients; Recormon $^{\oplus}$, Boehringer Mannheim, Rotkreuz, Switzerland, in one patient) was given intravenously at the end of the dialysis sessions in two patients and subcutaneously in the two other patients, three times weekly. Haematological parameters were assessed monthly; a clinical survey was regularly performed in all cases to detect any worsening of the multiple myeloma. Values are given as mean $( \pm \mathrm{SD})$.

\section{Results}

All patients presented at stage IIIB [2] of multiple myeloma and with IgG kappa serum $M$ component. The clinical and haematological characteristics of the patients before rHuEpo therapy are summarized in Table 1 . The last course of chemotherapy preceded the beginning of rHuEpo treatment by 4,5 , and 23 months in three cases, whereas the remaining patient did not receive any cancer treatment before starting rHuEpo. During the 6 months before $\mathrm{rHuEpo}$ treatment patients were given multiple blood transfusions. Plasma ferritin levels were more than $1000 \mu \mathrm{g} / \mathrm{l}$ in three cases and $403 \mu \mathrm{g} / \mathrm{l}$ in the last patient before rHuEpo. The modes of rHuEpo therapy and haematological response to treatment are described in Table 2. Mean haematocrit increased from $23 \pm 3$ to $32 \pm 4 \%$ after the first month of rHuEpo therapy; during the same time rHuEpo 

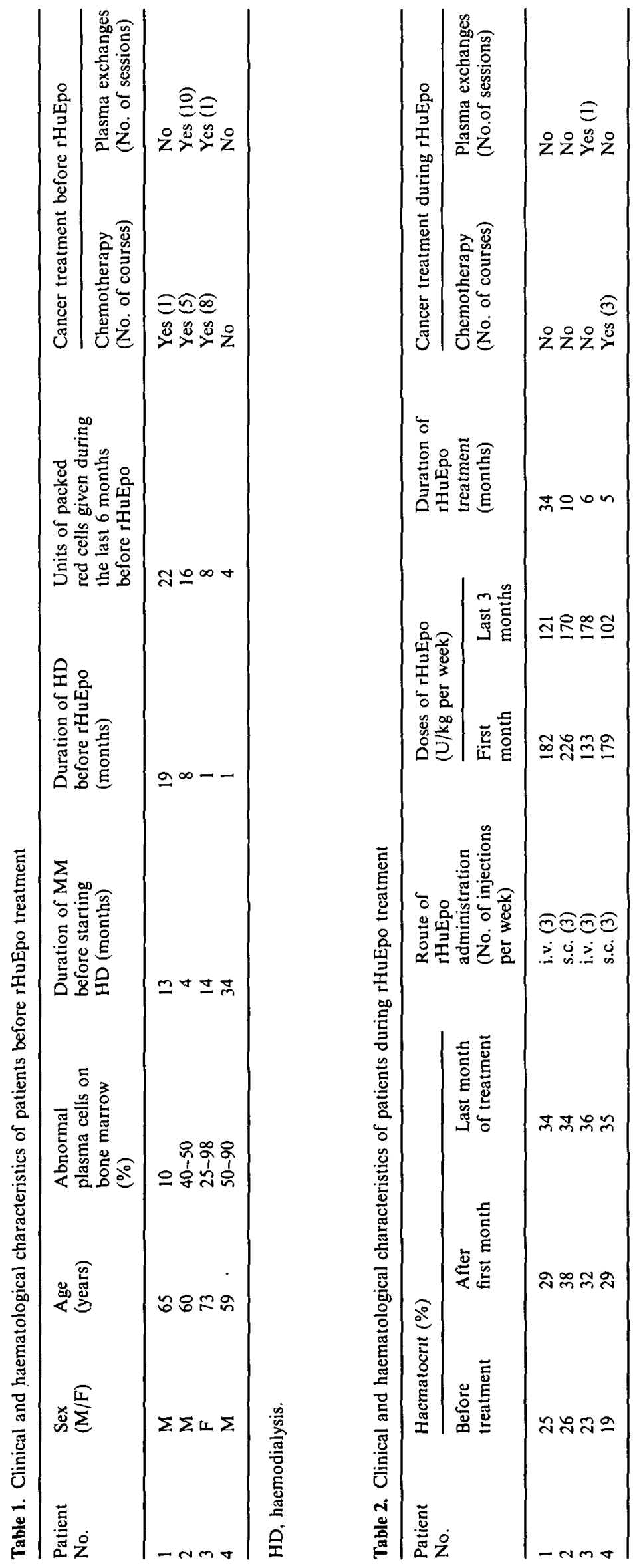
doses were $180 \pm 38 \mathrm{U} / \mathrm{kg}$ per week. The cumulative duration of treatment with rHuEpo was 55 months. Doses of rHuEpo during the last 3 months studied were $143 \pm 37 \mathrm{U} / \mathrm{kg}$ per week and were comparable to the maintenance doses applied over the whole followup period. There was no longer a need for blood transfusions. All patients reported a rapid improvement in wellbeing. Figure 1 illustrates the evolution of anaemia before and during rHuEpo therapy in patient 1. During rHuEpo treatment, antihypertensive therapy was started in one patient and the increase in blood pressure required larger doses of antihypertensive drugs in two other cases.

In the first case a second bone marrow biopsy, performed 25 months after the beginning of $\mathrm{rHuEpo}$, disclosed only $11 \%$ of abnormal plasma cells; serum IgG was $23.5 \mathrm{~g} / \mathrm{l}$ before $\mathrm{rHuEpo}$ and remained stable, around $30 \mathrm{~g} / \mathrm{l}$, during the 30 months following rHuEpo introduction. In the second patient, serum $\operatorname{IgG}$ was $29.5 \mathrm{~g} / \mathrm{l}$ before and $9.2 \mathrm{~g} / \mathrm{l}$ just after chemotherapy and plasma exchanges; after 10 months of treatment with rHuEpo, serum IgG was $17.4 \mathrm{~g} / \mathrm{l}$, whereas cancer treatment had been discontinued for more than 1 year. In the third patient, serum IgG levels of $101 \mathrm{~g} / 1$ decreased to $51 \mathrm{~g} / \mathrm{l}$ after a course of chemotherapy (vincristine, adriamycin, and dexamethasone). Following an exacerbation of the disease, chemotherapy was restarted and linked to plasma exchanges. Six months after the beginning of rHuEpo and 3 months after the last plasma exchanges, serum IgG was $42.2 \mathrm{~g} / \mathrm{l}$. Finally, after chemotherapy the last patient recovered a sufficient level of renal function to stop haemodialysis. rHuEpo was given for 5 months, until the anaemia resolved spontaneously. The light chains were no longer detectable in blood and urine at the end of
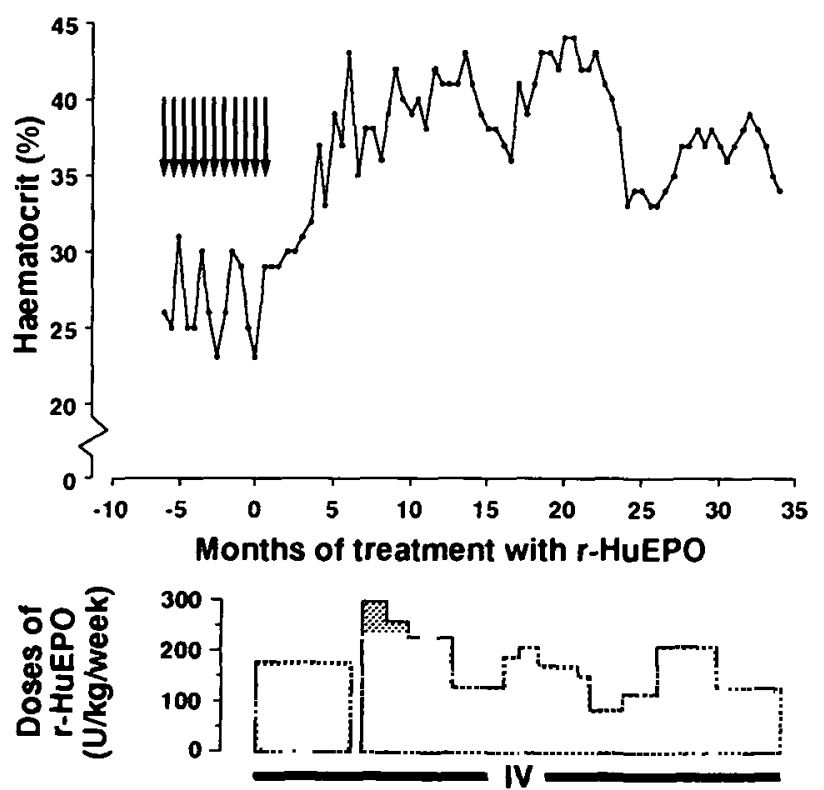

Fig. 1. Changes in haematocrit in patient 1 before and during rHuEpo therapy. Each arrow represents the transfusion of two packed red-cells. Information on the pretreatment period was retrospectively collected during the 6 months preceding rHuEpo therapy.
rHuEpo administration. There were no episodes of severe hypercalcaemia in any patient, and clinical examination disclosed no aggravation of the disease during rHuEpo therapy.

\section{Discussion}

Renal failure is generally considered to be an ominous complication in patients with multiple myeloma [3]. Despite the fact that end-stage renal disease is considered an indication of a poor prognosis in multiple myeloma, long-term survival has been observed in patients requiring renal replacement therapy [4]. Thus it seems evident that in those patients anaemia must also be corrected without the hazards inherent in blood transfusions. The successful administration of $\mathrm{rHuEpo}$ to two patients on regular haemodialysis for end-stage renal disease due to multiple myeloma has been reported recently [5]; the duration of the follow up on rHuEpo treatment was short in these cases. More recently, Holley et al. published the case of a patient on continuous peritoneal dialysis who was treated successfully with rHuEpo, but information about the evolution of myeloma during the 7-month period of treatment was lacking [6]. These publications followed the report of erythropoietin treatment of anaemia associated with multiple myeloma in patients without renal failure [1], which did not show any interaction between the underlying disease and the response to erythropoietin therapy after 6 months treatment. In this paper Ludwig et al. reported an $85 \%$ success rate in the correction of anaemia [1]. In the present study all patients responded dramatically to the treatment with rHuEpo. Mean doses of $\mathrm{rHuEpo}$ remained relatively stable throughout the study period and comparable to the doses administered to our group of haemodialysed patients without malignancy $(143 \pm 37$ and $134 \pm 56 \mathrm{U} / \mathrm{kg}$ per week, respectively) [7]. Since renal function was moderately to severely depressed at the time of diagnosis of multiple myeloma in two patients, anaemia might be mainly the consequence of decreased production of endogenous erythropoietin, rather than the direct effet of multiple myeloma on the bone marrow. Nevertheless, the degree of anaemia was greater than expected at that time to be attributed solely to renal failure. Furthermore, in the patients 3 and 4 anaemia was already present before the onset of renal failure. Thus it seems reasonable to attribute the cause of anaemia to both renal and haematological problems during rHuEpo therapy.

Treatment with rHuEpo produced an obvious improvement in quality of life and ended the need for blood transfusions. Clinical and haematological monitoring did not disclose a stimulatory effect of $\mathrm{rHuEpo}$ on the malignant clone over the study period ranging from 5 to 34 months. Conversely, the activity of the disease remained stable, despite abstention from chemotherapy by three patients, during that time, when taking into account the changes in IgG levels and the degree of bone marrow infiltration by abnormal plasma 
cells when available. This is at odds with the case reported by Rogers et al., which exhibited a temporary increase in urinary excretion of lambda light chains, reversed to the previous level after cessation of treatment with rHuEpo [8]. It would in theory be surprising if $\mathrm{rHuEpo}$ had favoured myeloma growth because there is no evidence of a stimulatory effect of this substance on B lineage cells, even though some authors have shown effects on non-erythroid haemopoietic progenitor cells $[9,10]$. On the contrary, the rather quiet course of the myeloma raises the possibility that avoiding blood transfusions might have decreased the growth rate of the aberrant B cell clone. Effectively, the immunosuppressive nature of blood transfusions may decrease non-specific immune response and thus further enhance the susceptibility of patients with myeloma to infectious complications $[11,12]$ and perhaps also the rate of tumour growth [12]. Such a hypothesis, however, needs confirmation. We conclude that in our experience rHuEpo treatment in haemodialysed patients suffering from multiple myeloma is safe, well tolerated, and as effective as in patients on haemodialysis without malignancy.

Acknowledgements. Parts of this work were presented at the Annual Meeting of the Société de Néphrologie, Genève, Switzerland, October 1991, and were published in abstract form in Néphrologie 13: 55, 1992. This clinical research was supported by grants from Cilag, Schaffhausen, Switzerland and The Elie Safra Medical Fund. We are grateful to the nursing staff of the Dialysis Centre of Geneva for assistance, and to Miss $\mathbf{J}$. Bayley for her valuable review of the manuscript.

\section{References}

1. Ludwig H, Fritz E, Kotzmann H, Hocker P, Gisslinger $\mathbf{H}$, Barnas U. Erythropoietin treatment of anemia associated with multiple myeloma. $N$ Engl J Med 1990; 322: 1693-1699

2. Durie BG, Salmon SE. A clinical staging system for multiple myeloma: correlation of measured myeloma cell mass with presenting features, response to treatment, and survival. Cancer 1975; 36: 842-854

3. Alexanian R, Balcerzak S, Bonnet JD et al. Prognostıc factors in multiple myeloma. Cancer 1975; 36: 1192-1201

4. Korzets A, Tam F, Russel G, Feehally J, Walls J. The role of continuous ambulatory peritoneal dialysis in end-stage renal failure due to multiple myeloma. Am J Kidney Dis 1990; 26 : 216-223

5. Taylor J, Mactier RA, Stewart WK, Henderson IS. Effect of erythropoietin on anaemia in patients with myeloma receiving haemodialysis. Br Med J 1990; 301: 476-477

6. Holley JL, Nolan TA, Piraino B. Recombinant human erythropoietın in a patient with multiple myeloma and end-stage renal disease. Clin Nephrol 1992; 37: 145-147

7. Ruedin P, Pechère Bertschi A, Stoermann C, Leski M. Comparison de l'efficacité et de la tolérance de l'érythropoïétine humaine recombinante entre l'administration intra-veineuse et souscutanée en hémodialyse chronıque. Néphrologie 1992; 13: 87-92

8. Rogers S, Russel NH, Morgan AG. Effect of erythropoietin in patients with myeloma. $\mathrm{Br}$ Med J 1990; 301: 667

9. Dessypris E, Graber SE, Krantz SB, Stone WJ. Effects of recombinant erythropoietin on the concentration and cycling status of human marrow hematopoietic progenitor cells in vivo. Blood 1988; 72: 2060-2062

10. Geissler K, Stockenhuber F, Kabrna E, Hinterberger W, Balcke $P$, Lechner $K$. Recombinant human erythropoietın and hematopoietic progenitor cells in vitro. Blood 1989; 73: 2229

11. Louis F, Ruedin P, Frei D, Jeannet M, Favre H. Importance of the role of blood transfusions in delayed hypersensitivity among end-stage renal disease patients. Proc Eur Dial Transplant Assoc 1985; 22: 937-940

12. Waymack JP, Alexander JW. Blood transfusions as an immunomodulator-A review. Comp Immunol Microbiol Infect Dis 1986; 9: $177-183$

Received for publication 29.7 .92

Accepted in revised form 22.10 .92 